\title{
Impact of working conditions on the health of pharmacy workers
}

\author{
Irina Filina*, Svetlana Nikishina, Irina Snimshchikova, Anastasiia Kulakova, Anastasiia Ovchinnikova, and Ilya \\ Leonidov
}

Medical Institute, Oryol State University named after I.S. Turgenev, Oryol, Russia

\begin{abstract}
The analysis revealed factors that have an adverse effect on human health: artificial lighting (I $=0.72)$; standing activity $(\mathrm{I}=0.63)$; monotonous movements $(\mathrm{I}=0.56)$; prolonged work at the computer $(\mathrm{I}$ $=0.55)$. It was found that many pharmacy workers have chronic diseases: vascular pathology $(29.4 \%)$, musculoskeletal pathology (28.4\%), gastrointestinal tract pathology (27.5\%); visual impairment (27.5\%). It was found that $20.6 \%$ of pharmacy employees do not have breaks for rest and meal; $13.7 \%$ do not have the opportunity to take warm food at work; $35.3 \%$ have the biggest meal of the day in the evening. In the workplace, a set of factors that have an adverse effect on the health of pharmaceutical professionals has been identified; many workers have work-related chronic diseases; most pharmacists experience discomfort after work; some employers violate labour legislation by not providing specialists with breaks for rest and meal.
\end{abstract}

\section{Introduction}

The work of a pharmaceutical specialist in a pharmacy is one of the most difficult and stressful types of work. In the process of dispensing and manufacturing medicines in a pharmacy, employees may be adversely affected by the factors of the production environment: dust, vapors, gases from medicines and reagents; noise; microclimate of the room; microbial contamination; visual strain; uncomfortable working posture, etc. [1-4].

A heavy responsibility, greater focus, and conflicts with visitors lead to a psychological and emotional overload [3]. Therefore, the purpose of our study was to examine the impact of working conditions on the health of pharmacy workers.

\section{Experimental procedure}

In the process of solving the tasks, marketing and factor analysis as well as sociological methods were used. Database of the study was formed on the basis of a survey, 102 pharmaceutical specialists of pharmaceutical organizations of the Oryol region took part in the survey, including $64(62.7 \%)$ pharmacists and 38 (37.3\%) pharmacy technicians. The sample size required for the sociological research was calculated using (1):

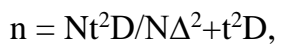

where $\mathrm{N}$-the general population, representing the number of pharmaceutical specialists in the Oryol region in 2019 (according to the data from the Department of Health of the Oryol region); $\Delta$-the margin of error of the sample rate, which equals 0.05-0.1; D-variance, which equals 0.25 ; t-Student's t-distribution coefficient, which equals 1.96 (if $\mathrm{p}=0.95$ ).

$$
\mathrm{n}=1980 * 1,96^{2} * 0,25 / 1980 * 0,1^{2}+1,96^{2 *} 0,25=91,6
$$

The questionnaire contains the following sections: socio-demographic characteristics of respondents; work and rest schedule; characteristics of work activity. The questionnaire also includes a list of factors that have an adverse effect on the health of pharmacy employees. Qualitative characteristics were used as criteria for evaluating results, each of qualitative characteristics was assigned a corresponding numerical value-coefficient $[5,6]$.

The index for each of the factors was calculated using the formula:

$$
\mathrm{I}=\frac{1.0 * \mathrm{n} 1+0.5 * \mathrm{n} 2+(-0.5) * \mathrm{n} 3+(-1.0) * \mathrm{n} 4}{\mathrm{n} 1+\mathrm{n} 2+\mathrm{n} 3+\mathrm{n} 4},
$$

where $n 1, n 2, n 3, n 4$ - the corresponding number of respondents combined by one of the four possible responses on the satisfaction scale.

Statistical processing of the results was performed using the Microsoft Excel software package. The data is presented as an absolute and relative (\%) number of pharmaceutical specialists.

\section{Results and Discussion}

In order to study the working conditions of pharmaceutical specialists, a survey was conducted among employees of pharmacy organizations in the Oryol region, $91(89.2 \%)$ of the respondents were women and $11(10.8 \%)$ were men.

The average age of the respondents was $40 \pm 20$ years. $64(62.7 \%)$ specialists with higher professional 
education and $38(37.3 \%)$ with secondary vocational education took part in the survey. $26(25.5 \%)$ of the survey participants work in state-owned pharmacies; 33 (32.4\%) work in municipal pharmacies; and 43 (42.1\%) kiosk; $8(7.8 \%)$ - the position of a deputy head of a pharmacy; $4(3.9 \%)$ - the position of a department supervisor; 40 (39.2\%) - the position of a pharmacist; 36 $(35.34 \%)$ - the position of a pharmacy technician. 46 (45.1\%) pharmaceutical specialists have less than 5 years of specialized work experience; 27 (26.5\%) - 6 to 15 years; $7(6.9 \%)$ - 16 to 25 years; $13(12.7 \%)$ - from 25 to 35 years; $9(8.8 \%)$ - over 35 years.

In the course of the study, it was found that 51 $(50.0 \%)$ of the respondents work every day for 6-8 hours, this number of working hours is typical for municipal and state pharmacies.

In private pharmacy organizations the typical work schedule is two days of work followed by two days of rest, this is the way $42(41.2) \%$ of respondents work. It was found out that $9(8.8 \%)$ pharmaceutical specialists work more than 12 hours a day. $2(1.96 \%)$ respondents have night shifts; 3 (2.94\%) - work during off-hours.

The survey revealed that $3(2.94 \%)$ pharmaceutical specialists start a 12-hour shift immediately after the night shift. Taking into account that 42 (41.2\%) respondents indicated that their work schedule has a negative impact on their family life, we can conclude that the work schedule in many pharmacy organizations is irrational.

When questioned, pharmaceutical specialists complained that it is physically difficult to work 12 hours a day, that they do not have time to do household chores and take good care of children.

It was found that the majority of respondents - 62 $(60.8 \%)$ - work 5 days a week. In private pharmacies, pharmaceutical specialists - 27 (26.44\%) - work 4 days a week, since they typically work for two days and then rest for another two days. It is alarming that $11(10.8 \%)$ respondents sometimes work 6 days a week and 2 people (1.96\%) - 7 days a week.

In the course of the study, it was found that in the compounding pharmacies the most unfavourable factors of the indoor environment include the direct impact of drug substances on the pharmacist in the process of manufacturing drugs.

Moreover, harmful substances may be released into the air during intra-pharmaceutical prepackaging of dosage forms. In case of a violation of the sanitary and hygienic conditions of the technological process and poor personal hygiene, medicinal substances in the form of dust or aerosols can enter the body of workers through the lungs, skin and mucous membrane. At the same time, vapours of volatile substances can get in the air: formalin, ammonia, acetic acid, ethyl alcohol, camphor, menthol and other substances in concentrations exceeding the maximum permissible concentration.

Medicinal dust can be harmful to the pharmacist. Many types of medicinal dust have an adverse effect on the mucous membrane of the upper respiratory tract of a person during industrial contact, for example, dust of zinc oxide, talc, salicylic acid. Substances that have a pronounced irritant effect and often cause allergic reactions include nicotinic acid, iodine.

Pharmacy workers may be prone to more severe reactions than patients who use them, since they can work in private pharmacies, 2 of the respondents $(1.96 \%)$ hold the position of a director of a pharmacy chain; $12(11.8 \%)$ - the position of a head of a pharmacy, pharmacy branch or pharmacy receive a dose during the working day that significantly exceeds the daily therapeutic dose during treatment.

It has been established that, in addition to medicinal substances, chemical reagents also have a harmful effect on pharmacists-analysts, which specialists use when conducting qualitative and quantitative analyses. Continuous inhalation of vapours of hydrochloric, sulphuric, acetic acids, alkalis and other substances can lead to serious illnesses.

According to the Labour Code of the Russian Federation, workers who are employed in jobs with harmful or hazardous working conditions, should have reduced working time that does not exceed 36 hours per week.

Workplace factors are considered harmful if their impact can lead to illness of an employee, and hazardous are those factors, the impact of which can lead to injury. In order to identify harmful and hazardous workplace factors, certification of workplaces is carried out. All employers are obliged to carry out certification of workplaces.

The list of categories of medical workers who are entitled to reduced working hours due to the work in hazardous conditions is given in section XL "Healthcare" of the List. In accordance with paragraph 137 of this section, pharmacists and pharmacy technicians of a pharmacy, except for those engaged exclusively in dispensing drugs without prescriptions and other goods of the pharmacy range, are entitled to reduced working time of 36 hours per week.

When questioned, pharmaceutical specialists in the surveyed pharmacies, it was found that almost a third of the respondents - $27(26.5 \%)$ - do not know what assessment of workplaces in terms of working conditions is and when was the last time they had it in a pharmacy organization.

The study found that the majority of pharmacy workers - $85(83.3 \%)$ - believe that their workplace is planned rationally. However, the survey revealed that only $38(37.3 \%)$ respondents have furniture with a rotating device at the workplace; the state of the microclimate and the level of illumination do not suit 22 $(21.6 \%)$ pharmaceutical specialists; 37 (36.3\%) people do not have a rest-room in their pharmacy.

The study analyzed some of the factors that have an adverse effect on the health of pharmacy employees (table I).

The entire set of factors was divided into two blocks: factors that have an adverse effect on the health of pharmaceutical professionals; the consequences of these factors. In the first block the respondents identified the most adverse factors: artificial lighting ( $\mathrm{I}=0.72$ ); standing activity ( $\mathrm{I}=0.63$ ); monotonous movements ( $\mathrm{I}$ $=0.56) ;$ prolonged work at the computer $(\mathrm{I}=0.55)$. In the second block, respondents determined their state of health at the end of the working day, as a result of which it turned out that many often feel: leg weakness (I $=0.58$ ); fatigue due to a large number of visitors (I $=0.55)$; moral responsibility for the patient $(\mathrm{I}=0.53)$; decrement in visual acuity and ocular discomfort (I $=0.41$ ). 
Table 1. Summary table of indices that characterize the degree of adverse effects of certain factors on the health of pharmaceutical professionals

\begin{tabular}{|c|l|c|}
\hline № & \multicolumn{1}{|c|}{ In relation to the factor } & I \\
\hline 1 & Standing activity & 0,63 \\
\hline 2 & Prolonged work at the computer & 0,55 \\
\hline 3 & Inhalation of harmful substances & $-0,42$ \\
\hline 4 & Psychological stress & 0,04 \\
\hline 5 & Monotonous movements & 0,56 \\
\hline 6 & Artificial lighting & 0,72 \\
\hline 7 & Contact with patients suffering from infectious diseases & 0,46 \\
\hline 8 & Inconvenient location of pharmacy furniture & $-0,3$ \\
\hline 9 & Leg weakness & 0,58 \\
\hline 10 & Fatigue due to the mental nature of the work & $-0,45$ \\
\hline 11 & Fatigue due to a large number of visitors & 0,55 \\
\hline 12 & Neurosis due to conflicts with visitors & 0,05 \\
\hline 13 & Feeling of a moral responsibility for the patient & 0,53 \\
\hline 14 & Acute respiratory diseases & 0,07 \\
\hline 15 & Working despite feeling unwell & 0,29 \\
\hline 16 & Feeling of decrement in visual acuity and ocular discomfort when working at the computer & 0,41 \\
\hline & & \\
\hline
\end{tabular}

The study found that 75 (73.5\%) pharmaceutical professionals are willing to work when they feel unwell, without taking the opportunity to take a sick leave. Many pharmacy workers have chronic diseases (Fig. 1).

The figure shows that many people have vascular pathology 30 (29.4\%), in most cases it is varicose veins; musculoskeletal pathology 29 (28.4\%), for example, arthrosis, arthritis. At the same time, the survey found that only $13(12.8 \%)$ of respondents regularly engage in industrial gymnastics. Visual impairment is present in 28 $(27.5 \%)$ of the respondents, but only $18(17.6 \%)$ of the respondents do eye exercises during the working day.

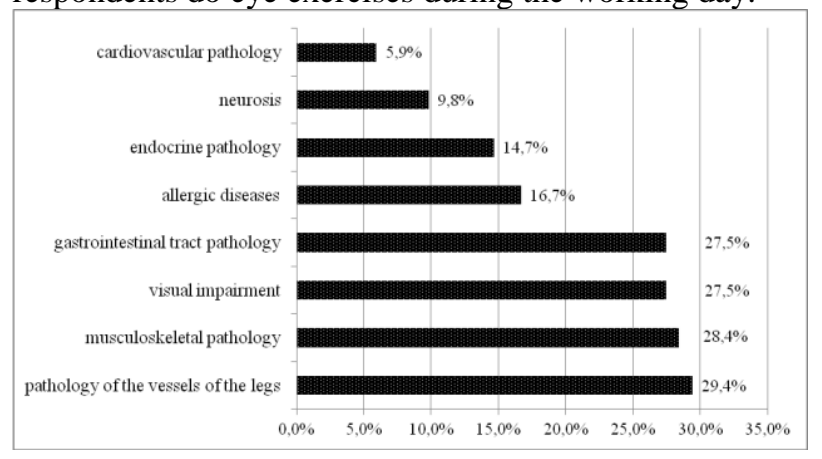

Fig. 1 Presence of chronic diseases in respondents

The analysis of questionnaires revealed that 30 (29.4\%) of respondents' experience heaviness in the legs, cramps in the calf muscles, swelling of the lower extremities in the evening or at night; 27 (26.5\%) of people experience undue fatigability after work (Fig. 2).

The study found that $28(27.5 \%)$ pharmaceutical specialists have gastrointestinal tract pathologies, and 65 (63.7\%) respondents have digestive problems in the form of nausea, heartburn, belching, flatulence, bloating, and abdominal pain. Article 108 of the Labour Code of the Russian Federation states that "during the working day (shift) the employee should be given a break for rest and meal of at least 30 minutes, which is not included into working hours.

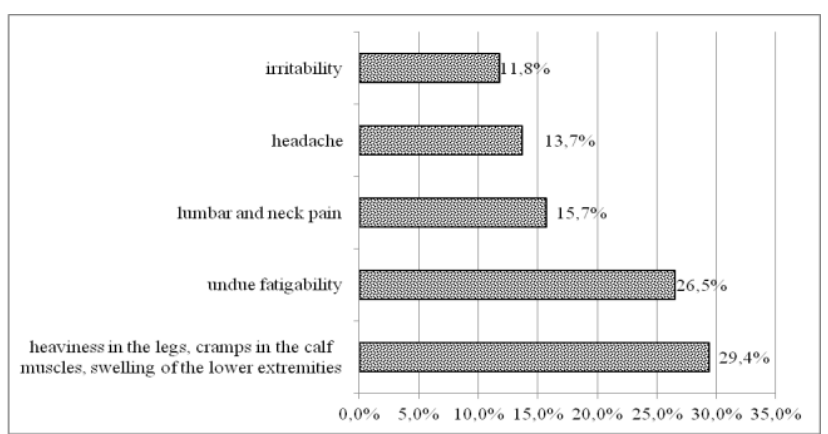

Fig. 2 Discomfort of the respondents after work

The time of a break and its concrete duration are established by the internal labour regulations or on agreement between the employee and the employer. In kinds of work where, according to the conditions of production (work), it is impossible to give a break for rest and meal, the employer must give the employee an opportunity to have rest and meal during the working hours. The list of such kinds of work as well as places for rest and meals are established by the internal labour regulations [2]. The survey revealed that 21 (20.6\%) of pharmacy employees do not have a break for rest and meal; $14(13.7 \%)$ of respondents do not have the opportunity to take warm food at work. It was found that $16(15.7 \%)$ of the respondents take the biggest meal of the day in the morning; $50(49.0 \%)$ at lunch and 36 $(35.3 \%)$ in the evening. Therefore, it is not surprising that $31(30.4 \%)$ of pharmaceutical specialists are overweight. It was found that the majority of pharmacists and pharmacy technicians 99 (97\%) visit a doctor at least once a year, but only $23(22.5 \%)$ of the respondents regularly undergo annual physical examination. 


\section{Conclusions}

Thus, in the course of the study

- a set of factors that have an adverse effect on the health of pharmaceutical specialists in the workplace has been identified;

- many pharmacists and pharmacy technicians have work-related chronic diseases;

- most pharmacy workers experience discomfort after work;

- some employers violate labour laws by not providing specialists with a break for rest and meal.

\section{References}

1 A. A. Svechkova, R. V. Shaigorodsky, "Influence of the nature of work and working conditions on the performance and health of employees of pharmacy organizations," Current issues of modern medicine. Collection of materials of the I Far Eastern medical youth forum. (publishing house of DVSMU, Khabarovsk, p. 272-273, 2017)
2 Labour code of the Russian Federation: Federal law of 30.12.2001 N 197-FZ (revision of 24.04.2020). Electronic legal reference system "Consultant plus". Access code: http://www.consultant.ru . Date of access 03.06.2020.

3 I. A. Filina, A. Yu. Ovchinnikova, and A. S. Kolesnikov, "Professional stresses in pharmaceutical specialists," Innovations in life sciences. Collection of materials of the II International Symposium may p. 19-20, Belgorod, (2020), 320-322.

4 I. A. Filina, I. M. Razdorskaya, J. Scientific Bulletin of the Belgorod state University, 12, 159-168, (2016)

5 Allmendinger, M. Emmerich, and J. Hakanen, J. of Multi-Criteria Decision Analysis, 24, 5-24, (2017)

6 Jason W. OsborneJ. Practical Assessment, Research and Evaluation, 20, 1-6, (2015) 